\title{
REFINED BOHR-TYPE INEQUALITIES WITH AREA MEASURE FOR BOUNDED ANALYTIC FUNCTIONS
}

\author{
YONG HUANG, MING-SHENG LIU *, AND SAMINATHAN PONNUSAMY
}

\begin{abstract}
In this paper, we establish five new sharp versions of Bohr-type inequalities for bounded analytic functions in the unit disk by allowing Schwarz function in place of the initial coefficients in the power series representations of the functions involved and thereby, we generalize several related results of earlier authors.
\end{abstract}

\section{Introduction and Preliminaries}

Let $\mathbb{D}:=\{z \in \mathbb{C}:|z|<1\}$ denote the open unit disk in $\mathbb{C}$. A remarkable discovery of Herald Bohr [10] in 1914 states that if $H_{\infty}$ denotes the class of all bounded analytic functions $f$ on $\mathbb{D}$ with the supremum norm $\|f\|_{\infty}:=\sup _{z \in \mathbb{D}}|f(z)|$, then

$$
B_{0}(f, r):=\left|a_{0}\right|+\sum_{n=1}^{\infty}\left|a_{n}\right| r^{n} \leq\|f\|_{\infty} \text { for } 0 \leq r \leq 1 / 6,
$$

where $a_{k}=f^{(k)}(0) / k$ ! for $k \geq 0$. Later M. Riesz, I. Shur and F. W. Wiener, independently proved its validity on a wider interval $0 \leq r \leq 1 / 3$, and the family of functions $\varphi_{a}(z)=$ $(a-z) /(1-\bar{a} z)(|a|<1)$ as $a \rightarrow 1$ demonstrates that the number $1 / 3$ is optimal. This result is usually referred to as Bohr's power series theorem for the unit disk and $1 / 3$ is called the Bohr radius. We refer the paper of Bohr [10] which contains the proof of Wiener showing that the Bohr radius is $1 / 3$. See also $[30,31]$ for other proofs. Then it is worth pointing out that there is no extremal function in $\mathcal{B}$ such that the Bohr radius is precisely 1/3 (cf. [7], [13, Corollary 8.26] and [14]). Several aspects of Bohr's inequality and its extensions in various settings may be seen in the literature. For example, the Bohr radius for analytic functions from the unit disk into different domains, such as the punctured unit disk or the exterior of the closed unit disk or concave wedge-domains, have been analyzied in $[1,2,3,4]$. Ali et al. $[6,14]$ considered the problem of determining Bohr radius for the classes of even and odd analytic functions and for alternating series. The articles $[7,19,24]$ concerned with the class of all sense-preserving harmonic mappings and the Bohr radius for sense-preserving harmonic quasiconformal mappings. Defant [11] improved a version of the Bohnenblust-Hille inequality, and in 2004, Paulsen [26] proved a uniform algebra analogue of the classical inequality of Bohr concerning Fourier coefficients of bounded holomorphic functions. In [25, 27], the authors demonstrated the classical Bohr inequality using different methods of operators. Djakov and Ramanujan [12] have established the results on Bohr's phenomena for multidimensional power series.

File: arXiv-LiuSamyHuang3_2020_.tex, printed: 14-9-2020, 0.52

2000 Mathematics Subject Classification. Primary: 30A10, 30C45, 30C62; Secondary: $30 \mathrm{C} 75$.

Key words and phrases. Bohr radius, bounded analytic functions, harmonic function, Bohr inequality

* Correspondence should be addressed to Ming-Sheng Liu . 
Recently, in [22, 23, 28, 29], the authors presented refined versions of Bohr's inequality along with few other related improved versions of previously known results. See also the recent survey articles $[5,17,18]$ and [13, Chapter 8]. Especially, after the appearance of the articles [5] and [15], several approaches and new problems on Bohr's inequality in the plane were investigated in the literature (cf. [8, 9, 16, 20, 22, 28, 29]).

One of our aims in this article is to generalize or improve recent versions of Bohr's inequalities for functions from $H_{\infty}$.

1.1. Basic Notations. Before we continue the discussion, we fix some notations. Throughout the discussion, we let

$$
\begin{aligned}
\mathcal{B} & =\left\{f \in H_{\infty}:\|f\|_{\infty} \leq 1\right\}, \text { and } m \in \mathbb{N}=\{1,2, \cdots\}, \\
\mathcal{B}_{m} & =\left\{\omega \in \mathcal{B}: \omega(0)=\cdots=\omega^{(m-1)}(0)=0 \text { and } \omega^{(m)}(0) \neq 0\right\} .
\end{aligned}
$$

Also, for $f(z)=\sum_{n=0}^{\infty} a_{n} z^{n} \in \mathcal{B}$ and $f_{0}(z):=f(z)-f(0)$, we let for convenience

$$
B_{k}(f, r):=\sum_{n=k}^{\infty}\left|a_{n}\right| r^{n} \text { for } k \geq 0, \text { and }\left\|f_{0}\right\|_{r}^{2}:=\sum_{n=1}^{\infty}\left|a_{n}\right|^{2} r^{2 n}
$$

so that $B_{0}(f, r)=\left|a_{0}\right|+B_{1}(f, r)$ and $B_{0}(f, r)=\left|a_{0}\right|+\left|a_{1}\right| r+B_{2}(f, r)$.

1.2. Refined Bohr's inequalities and basic problems. Recently, Ponnusamy et al. [28] proved the following refined Bohr inequality.

Theorem A. ([28]) Suppose that $f \in \mathcal{B}, f(z)=\sum_{n=0}^{\infty} a_{n} z^{n}$ and $a=\left|a_{0}\right|=|f(0)|$. Then

$$
B_{0}(f, r)+\frac{1+a r}{(1+a)(1-r)}\left\|f_{0}\right\|_{r}^{2} \leq 1 \text { for } r \leq \frac{1}{2+a}
$$

and the numbers $\frac{1}{2+a}$ and $\frac{1}{1+a}$ cannot be improved. Moreover,

$$
a^{2}+B_{1}(f, r)+\frac{1+a r}{(1+a)(1-r)}\left\|f_{0}\right\|_{r}^{2} \leq 1 \text { for } r \leq \frac{1}{2}
$$

and the numbers $\frac{1}{2}$ and $\frac{1}{1+a}$ cannot be improved.

Besides these results, there are plenty of works about the classical Bohr inequality. Based on the work of Kayumov and Ponnusamy [16], several forms of Bohr-type inequalities for the family $\mathcal{B}$ were obtained in [22] when the Taylor coefficients of classical Bohr inequality are partly or completely replaced by higher order derivatives of $f$. With the development of Bohr-type inequalities, the authors in [21] established improved version of the Bohr-Rogosinski inequality and considered some refined Bohr type inequalities associated with area, modulus of $f-a_{0}(f)$ and higher order derivatives of $f$ in part. Here we recall part of them.

Theorem B. ([21]) Suppose that $f \in \mathcal{B}, f(z)=\sum_{n=0}^{\infty} a_{n} z^{n}$ and $a:=\left|a_{0}\right|=|f(0)|$. Then

$$
|f(z)|+B_{1}(f, r)+\frac{1+a r}{(1+a)(1-r)}\left\|f_{0}\right\|_{r}^{2} \leq 1
$$


for $|z|=r \leq r_{a}=2 /(3+a+\sqrt{5}(1+a))$. Then the radius $r_{a}$ is best possible and $r_{a} \geq \sqrt{5}-2$. Moreover

$$
|f(z)|^{2}+B_{1}(f, r)+\frac{1+a r}{(1+a)(1-r)}\left\|f_{0}\right\|_{r}^{2} \leq 1
$$

for $|z|=r \leq r_{a}^{\prime}$, where $r_{a}^{\prime}$ is the unique positive root of the equation

$$
\left(1-a^{3}\right) r^{3}-(1+2 a) r^{2}-2 r+1=0 .
$$

The radius $r_{a}^{\prime}$ is best possible. Further, we have $1 / 3<r_{a}^{\prime}<1 /(2+|a|)$.

Theorem C. ([21]) Suppose that $f \in \mathcal{B}, f(z)=\sum_{n=0}^{\infty} a_{n} z^{n}$ and $a:=\left|a_{0}\right|=|f(0)|$. Then

$$
B_{0}(f, r)+\frac{1+a r}{(1+a)(1-r)}\left\|f_{0}\right\|_{r}^{2}+\left|f_{0}(z)\right| \leq 1
$$

for $|z|=r \leq \frac{1}{5}$ and the number $\frac{1}{5}$ cannot be improved. Moreover,

$$
a^{2}+B_{1}(f, r)+\frac{1+a r}{(1+a)(1-r)}\left\|f_{0}\right\|_{r}^{2}+\left|f_{0}(z)\right| \leq 1
$$

for $|z|=r \leq \frac{1}{3}$ and the constant $\frac{1}{3}$ cannot be improved.

Theorem D. ([21]) Suppose that $f \in \mathcal{B}, f(z)=\sum_{n=0}^{\infty} a_{n} z^{n}$ and $a:=\left|a_{0}\right|=|f(0)|$. Then

$$
|f(z)|+\left|f^{\prime}(z)\right| r+B_{2}(f, r)+\frac{1+a r}{(1+a)(1-r)}\left\|f_{0}\right\|_{r}^{2} \leq 1
$$

for $|z|=r \leq \frac{\sqrt{17}-3}{4}$ and the constant $\frac{\sqrt{17}-3}{4}$ is best possible.Moreover,

$$
|f(z)|^{2}+\left|f^{\prime}(z)\right| r+B_{2}(f, r)+\frac{1+a r}{(1+a)(1-r)}\left\|f_{0}\right\|_{r}^{2} \leq 1
$$

for $|z|=r \leq r_{0}$, where $r_{0} \approx 0.385795$ is the unique positive root of the equation

$$
1-2 r-r^{2}-r^{3}-r^{4}=0
$$

and the number $r_{0}$ is best possible.

Theorem E. ([21]) Suppose that $f \in \mathcal{B}, f(z)=\sum_{n=0}^{\infty} a_{n} z^{n}$ and $a:=\left|a_{0}\right|=|f(0)|$. Then for $|z|=r \leq \frac{1}{3}$,

$$
B_{0}(f, r)+\frac{1+a r}{(1+a)(1-r)}\left\|f_{0}\right\|_{r}^{2}+\left|f_{0}(z)\right|^{2} \leq 1
$$

is valid if and only if $0 \leq a \leq 4 \sqrt{2}-5 \approx 0.656854$.

It is natural to raise the following.

Problem 1. Whether we can further generalize or improve Theorems $B, C, D$ and $E$ ? 
In this article, we present an affirmative answer to this question in five different forms.

The paper is organized as follows. In Section 2, we present statements of five theorems which improve several versions of Bohr's type inequalities for bounded analytic functions, and several remarks. In Section 3, we state and prove a couple of lemmas which are needed for the proofs of two theorems. In Section 4, we present the proofs of the main results.

\section{Statement of Main Results and Remarks}

We now state a generalization of Theorem B in a general setting.

Theorem 1. Suppose that $f(z)=\sum_{n=0}^{\infty} a_{n} z^{n} \in \mathcal{B}, a:=\left|a_{0}\right|$ and $\omega \in \mathcal{B}_{m}$ for some $m \in \mathbb{N}$. Then we have

$$
A_{f}(z):=|f(\omega(z))|+B_{1}(f, r)+\frac{1+a r}{(1+a)(1-r)}\left\|f_{0}\right\|_{r}^{2} \leq 1
$$

for $r \in\left[0, \alpha_{m}\right]$, where $\alpha_{m}$ is the unique root in $(0,1)$ of the equation

$$
(1-r)\left(1-r^{m}\right)-2 r\left(1+r^{m}\right)=0 .
$$

The constant $\alpha_{m}$ cannot be improved. Moreover,

$$
B_{f}(z):=|f(\omega(z))|^{2}+B_{1}(f, r)+\frac{1+a r}{(1+a)(1-r)}\left\|f_{0}\right\|_{r}^{2} \leq 1
$$

is valid for $r \in\left[0, \beta_{m}\right]$, where $\beta_{m}$ is the unique root in $(0,1)$ of the equation

$$
1-2 r-r^{m}=0 .
$$

The constant $\beta_{m}$ cannot be improved.

Remark 1. We mention now several useful remarks and some special cases.

(1) One can state each of the two radii in Theorem 1 as a function of $a$. In that case, $\alpha_{m}$ and $\beta_{m}$ should be replaced by $\alpha_{m, a}$ and $\beta_{m, a}$ which are in fact the unique roots in $(0,1)$ of the equation $A_{m}(a, r)=0$ and $B_{m}(a, r)=0$, respectively, where

$$
A_{m}(a, r)=(1-r)\left(1-r^{m}\right)-(1+a) r\left(1+a r^{m}\right)
$$

and

$$
B_{m}(a, r)=(1-r)\left(1-r^{2 m}\right)-r\left(1+a r^{m}\right)^{2} .
$$

(2) If we set $m=1$ and $\omega(z) \equiv z$ in Theorem 1 , then we get Theorem B.

(3) If we set $m=1$ in (2.1), then we get $\alpha_{1}=\sqrt{5}-2$.

(4) If we set $m=2$ in (2.1), then it reduces to $r^{3}+r^{2}+3 r-1=0$ which gives the root $\alpha_{2} \approx 0.295598$ in the interval $(0,1)$.

(5) If we allow $m \rightarrow \infty$ in (2.1) (with $\omega(z)=z^{m}$ in $\left.A_{f}(z)\right)$, then $|f(\omega(z))| \rightarrow|f(0)|$ and $\alpha_{\infty}=1 / 3$.

(6) The case $m=1$ in (2.2) gives the $\operatorname{root} \beta_{1}=1 / 3$.

(7) The case $m=2$ in (2.2) gives the $\operatorname{root} \beta_{2}=\sqrt{2}-1$.

(8) If we allow $m \rightarrow \infty$ in (2.2) (with $\omega(z)=z^{m}$ in $B_{f}(z)$ ), then $\beta_{\infty}=1 / 2$. 
Theorem 2. Suppose that $f(z)=\sum_{n=0}^{\infty} a_{n} z^{n} \in \mathcal{B}, a:=\left|a_{0}\right|$ and $\omega \in \mathcal{B}_{m}$ for some $m \in \mathbb{N}$. Then we have

$$
C_{f}(z):=B_{0}(f, r)+\frac{1+a r}{(1+a)(1-r)}\left\|f_{0}\right\|_{r}^{2}+\left|f(\omega(z))-a_{0}\right| \leq 1
$$

for $r \in\left[0, \zeta_{m}\right]$, where $\zeta_{m}$ is the unique root in $(0,1 / 3]$ of the equation

$$
r^{m}(3-5 r)+3 r-1=0,
$$

or equivalently, $3 r^{m}+2 \sum_{k=1}^{m} r^{k}-1=0$. The upper bound $\zeta_{m}$ cannot be improved.

Moreover,

$$
D_{f}(z):=\left|a_{0}\right|^{2}+B_{1}(f, r)+\frac{1+a r}{(1+a)(1-r)}\left\|f_{0}\right\|_{r}^{2}+\left|f(\omega(z))-a_{0}\right| \leq 1
$$

for $r \in\left[0, \eta_{m}\right]$, where $\eta_{m}$ is the unique root in $(0,1 / 2]$ of the equation

$$
r^{m}(2-3 r)+2 r-1=0,
$$

or equivalently, $2 r^{m}+\sum_{k=1}^{m} r^{k}-1=0$. The upper bound $\eta_{m}$ cannot be improved.

Remark 2. The following special cases are useful and important to mention.

(1) The case $m=1$ and $\omega(z) \equiv z$ in Theorem 2 gives Theorem C.

(2) The case $m=1$ in (2.3) gives the root $\zeta_{1}=1 / 5$.

(3) The case $m=2$ in (2.3) reduces to $-5 r^{3}+3 r^{2}+3 r-1=(1-r)\left(5 r^{2}+2 r-1\right)=0$ which gives the root $\zeta_{2}=\frac{\sqrt{6}-1}{5} \approx 0.289898$ in the interval $(0,1 / 3)$.

(4) If we allow $m \rightarrow \infty$ in (2.3) (with $\omega(z)=z^{m}$ in $C_{f}(z)$ ), then $\zeta_{\infty}=1 / 3$.

(5) The case $m=1$ in (2.4) gives the root $\eta_{1}=1 / 3$.

(6) The case $m=2$ in $(2.4)$ reduces to $(1-r)\left(3 r^{2}+r-1\right)=0$ which gives the root $\eta_{2}=\frac{\sqrt{13}-1}{6} \approx 0.434259$ in the interval $(0,1 / 2)$.

(7) If we allow $m \rightarrow \infty$ in (2.4) (with $\omega(z)=z^{m}$ in $D_{f}(z)$ ), then $\eta_{\infty}=1 / 2$.

In Table 1 , we include the values of $\alpha_{m}, \beta_{m}, \zeta_{m}$ and $\eta_{m}$ for certain values of $m \geq 3$.

Theorem 3. Suppose that $f(z)=\sum_{n=0}^{\infty} a_{n} z^{n} \in \mathcal{B}, a:=\left|a_{0}\right|$ and $\omega \in \mathcal{B}_{m}$. Then we have

$$
E_{f}(z):=|f(\omega(z))|+|\omega(z)|\left|f^{\prime}(\omega(z))\right|+B_{2}(f, r)+\frac{1+a r}{(1+a)(1-r)}\left\|f_{0}\right\|_{r}^{2} \leq 1
$$

for $r \in\left[0, \gamma_{m}\right]$, where $\gamma_{m}$ is the unique root in $(0,1)$ of the equation

$$
r^{m}\left(r^{m}+2\right)\left[2 r^{2}-r+1\right]+2 r^{2}+r-1=0 .
$$

The upper bound $\gamma_{m}$ cannot be improved. Moreover,

$$
F_{f}(z):=|f(\omega(z))|^{2}+|\omega(z)|\left|f^{\prime}(\omega(z))\right|+B_{2}(f, r)+\frac{1+a r}{(1+a)(1-r)}\left\|f_{0}\right\|_{r}^{2} \leq 1
$$

for $r \in\left[0, \delta_{m}\right]$, where $\delta_{m}$ is the unique root in $(0,1)$ of the equation

$$
r^{m}\left(r^{m}+1\right)\left[r^{m}-r+2\right]+r-1=0 .
$$

The upper bound $\delta_{m}$ cannot be improved. 


\begin{tabular}{|l||l|l||l|l|}
\hline$m$ & $\alpha_{m}$ & $\beta_{m}$ & $\zeta_{m}$ & $\eta_{m}$ \\
\hline 3 & 0.319053 & 0.453398 & 0.318201 & 0.469396 \\
\hline 4 & 0.328197 & 0.474627 & 0.328083 & 0.484925 \\
\hline 5 & 0.331555 & 0.486389 & 0.331541 & 0.492432 \\
\hline 6 & 0.332731 & 0.492836 & 0.332729 & 0.496184 \\
\hline 7 & 0.333131 & 0.496292 & 0.333131 & 0.498077 \\
\hline 8 & 0.333266 & 0.498105 & 0.333266 & 0.499033 \\
\hline 9 & 0.333311 & 0.499040 & 0.333311 & 0.499515 \\
\hline 10 & 0.333326 & 0.499516 & 0.333326 & 0.499757 \\
\hline 15 & 0.333333 & 0.499985 & 0.333333 & 0.499992 \\
\hline 20 & 0.333333 & 0.500000 & 0.333333 & 0.500000 \\
\hline 25 & 0.333333 & 0.500000 & 0.333333 & 0.500000 \\
\hline 30 & 0.333333 & 0.500000 & 0.333333 & 0.500000 \\
\hline
\end{tabular}

TABLE 1. Numbers $\alpha_{m}, \beta_{m}, \zeta_{m}$ and $\eta_{m}$ are the unique roots in $(0,1)$ of the equations (2.1), (2.2), (2.3) and (2.4), respectively.

Theorem 4. Suppose that $f(z)=\sum_{n=0}^{\infty} a_{n} z^{n} \in \mathcal{B}, a:=\left|a_{0}\right|$ and $\omega \in \mathcal{B}_{m}$. Then we have

$$
G_{f}(z):=|f(\omega(z))|+|z|\left|f^{\prime}(\omega(z))\right|+B_{2}(f, r)+\frac{1+a r}{(1+a)(1-r)}\left\|f_{0}\right\|_{r}^{2} \leq 1
$$

for $r \in\left[0, \theta_{m}\right]$, where $\theta_{m}$ is the unique root in $(0,1)$ of the equation

$$
2 r^{2 m+2}-r^{2 m+1}+r^{2 m}+4 r^{m+2}+3 r-1=0 .
$$

The upper bound $\theta_{m}$ cannot be improved. Moreover,

$$
H_{f}(z):=|f(\omega(z))|^{2}+|z|\left|f^{\prime}(\omega(z))\right|+B_{2}(f, r)+\frac{1+a r}{(1+a)(1-r)}\left\|f_{0}\right\|_{r}^{2} \leq 1
$$

for $r \in\left[0, \vartheta_{m}\right]$, where $\vartheta_{m}$ is the unique root in $(0,1)$ of the equation

$$
r^{2 m+2}-r^{2 m+1}+r^{2 m}+2 r^{m+2}+2 r-1=0 .
$$

The upper bound $\vartheta_{m}$ cannot be improved.

Remark 3. Obviously, if we set $m=1$ and $\omega(z) \equiv z$ in Theorem 3 or Theorem 4 , then we get Theorem D.

In Table 2, we include the values of $\gamma_{m}, \delta_{m}, \theta_{m}$ and $\vartheta_{m}$ for certain values of $m \geq 2$.

Theorem 5. Suppose that $f(z)=\sum_{n=0}^{\infty} a_{n} z^{n} \in \mathcal{B}, a:=\left|a_{0}\right|$ and $\omega \in \mathcal{B}_{m}$ for some $m \geq 1$. We have the following:

(1) If $m=1$, then we have

$$
I_{f}(z):=B_{0}(f, r)+\frac{1+a r}{(1+a)(1-r)}\left\|f_{0}\right\|_{r}^{2}+\left|f(\omega(z))-a_{0}\right|^{2} \leq 1 \quad \text { for }|z|=r \leq 1 / 3
$$

if and only if $0 \leq a \leq a^{*}=-5+4 \sqrt{2} \approx 0.656854$. The constant $1 / 3$ cannot be improved.

(2) If $m \geq 2$, then (2.9) holds, and the constant $1 / 3$ cannot be improved. 


\begin{tabular}{|l||l|l||l|l|}
\hline$m$ & $\gamma_{m}$ & $\delta_{m}$ & $\theta_{m}$ & $\vartheta_{m}$ \\
\hline 2 & 0.391490 & 0.486848 & 0.316912 & 0.445688 \\
\hline 3 & 0.441112 & 0.535687 & 0.327911 & 0.472325 \\
\hline 4 & 0.467644 & 0.564540 & 0.331520 & 0.485708 \\
\hline 5 & 0.482442 & 0.582935 & 0.332726 & 0.492642 \\
\hline 6 & 0.490660 & 0.595034 & 0.333131 & 0.496239 \\
\hline 7 & 0.495127 & 0.603062 & 0.333266 & 0.498091 \\
\hline 8 & 0.497496 & 0.608373 & 0.333311 & 0.499037 \\
\hline 9 & 0.498727 & 0.611827 & 0.333326 & 0.499515 \\
\hline 10 & 0.499357 & 0.614117 & 0.333331 & 0.499757 \\
\hline 15 & 0.499980 & 0.617662 & 0.333333 & 0.499992 \\
\hline 20 & 0.500000 & 0.618000 & 0.333333 & 0.500000 \\
\hline 25 & 0.500000 & 0.618031 & 0.333333 & 0.500000 \\
\hline 30 & 0.500000 & 0.618034 & 0.333333 & 0.500000 \\
\hline
\end{tabular}

TABLE 2. Numbers $\gamma_{m}, \delta_{m}, \theta_{m}$ and $\vartheta_{m}$ are the unique roots in $(0,1)$ of the equations (2.5), (2.6), (2.7) and (2.8), respectively.

Remark 4. Obviously, the case $m=1$ and $\omega(z) \equiv z$ of Theorem 5 gives Theorem E.

\section{Key LEMmas AND THEIR PROOFS}

In order to establish our main results, we need the several lemmas which play key role in proving the subsequent results in Section 4.

\subsection{Three known lemmas.}

Lemma 1. (Schwarz-Pick Lemma) Let $\varphi(z)$ be analytic and $|\varphi(z)|<1$ in the unit disk D. Then

$$
\frac{\left|\varphi\left(z_{1}\right)-\varphi\left(z_{2}\right)\right|}{\left|1-\overline{\varphi\left(z_{1}\right)} \varphi\left(z_{2}\right)\right|} \leq \frac{\left|z_{1}-z_{2}\right|}{\left|1-\overline{z_{1}} z_{2}\right|} \text { for } z_{1}, z_{2} \in \mathbb{D}
$$

and equality holds for distinct $z_{1}, z_{2} \in \mathbb{D}$ if and only if $\varphi$ is a Möbius transformation. Also,

$$
\left|\varphi^{\prime}(z)\right| \leq \frac{1-|\varphi(z)|^{2}}{1-|z|^{2}} \text { for } z \in \mathbb{D},
$$

and equality holds for some $z \in \mathbb{D}$ if and only if $f$ is a Möbius transformation.

Lemma 2. ([14]) Suppose that $f(z)=\sum_{n=0}^{\infty} a_{n} z^{n} \in \mathcal{B}$ and $a:=\left|a_{0}\right|$. Then we have

$$
\sum_{n=1}^{\infty}\left|a_{n}\right| r^{n} \leq \begin{cases}r \frac{1-a^{2}}{1-r a} & \text { for } a \geq r, \\ r \frac{\sqrt{1-a^{2}}}{\sqrt{1-r^{2}}} & \text { for } a<r .\end{cases}
$$


Lemma 3. ([28]) Suppose that $f(z)=\sum_{n=0}^{\infty} a_{n} z^{n} \in \mathcal{B}$ and $a:=\left|a_{0}\right|$ Then the following inequality holds:

$$
B_{1}(f, r)+\frac{1+a r}{(1+a)(1-r)}\left\|f_{0}\right\|_{r}^{2} \leq\left(1-a^{2}\right) \frac{r}{1-r} \text { for } r \in[0,1) .
$$

A general version of this lemma is proved in [21, Lemma 4]. In particular, the following inequality holds (the case $N=2$ in [21, Lemma 4]) under the hypothesis of Lemma 3:

$$
B_{2}(f, r)+\frac{1+a r}{(1+a)(1-r)}\left\|f_{0}\right\|_{r}^{2} \leq\left(1-a^{2}\right) \frac{r^{2}}{1-r} \text { for } r \in[0,1) .
$$

\subsection{Two key lemmas.}

Lemma 4. There is a unique positive root $\zeta_{m}$ in $(0,1 / 3)$ of the equation $(2.3)$, and $\zeta_{m}$ satisfies the inequality

$$
\zeta_{m}^{m}+\frac{\zeta_{m}}{1-\zeta_{m}}+\frac{\zeta_{m}^{m}}{\sqrt{1-\zeta_{m}^{2 m}}} \leq 1
$$

Proof. We first prove the uniqueness of the solution in $(0,1 / 3)$ of the equation $(2.3)$.

Let $g(r)=r^{m}(3-5 r)+3 r-1$. Then, we find that $g(0)=-1<0$ and $g(1 / 3)=\frac{4}{3^{m+1}}>0$. Also, for $r \in[0,1 / 3]$, we have

$$
g^{\prime}(r)=m r^{m-1}(3-5 r)+3-5 r^{m}>0,
$$

showing that $g(r)$ is an increasing function of $r$ in $[0,1 / 3]$, and thus, $g(r)=0$ has a unique root $\zeta_{m}$ in $(0,1 / 3)$.

Now we verify the inequality (3.2). In fact, by (2.3), we note that

$$
\zeta_{m}^{m}=\frac{1-3 \zeta_{m}}{3-5 \zeta_{m}}, \zeta_{m} \in(0,1 / 3)
$$

For convenience, we set $x=\zeta_{m}$ and using the last relation, we have

$$
\begin{aligned}
x^{m}+\frac{x}{1-x}+\frac{x^{m}}{\sqrt{1-x^{2 m}}} & =\frac{1-3 x}{3-5 x}\left(1+\frac{1}{\sqrt{1-\left(\frac{1-3 x}{3-5 x}\right)^{2}}}\right)+\frac{x}{1-x} \\
& \leq \frac{1-3 x}{3-5 x}\left(1+\frac{1}{\sqrt{1-\left(\frac{1}{3}\right)^{2}}}\right)+\frac{x}{1-x} \\
& =\frac{4+3 \sqrt{2}}{4} \cdot \frac{1-3 x}{3-5 x}+\frac{x}{1-x} \\
& =\frac{(9 \sqrt{2}-8) x^{2}-(12 \sqrt{2}+4) x+4+3 \sqrt{2}}{4(3-5 x)(1-x)},
\end{aligned}
$$

which is less than or equal to 1 if $G(x) \geq 0$, where

$$
G(x)=(28-9 \sqrt{2}) x^{2}+(12 \sqrt{2}-28) x+8-3 \sqrt{2} .
$$

Since the discriminant of the equation $G(x)=0$ is less than 0 and $G(0)=8-3 \sqrt{2}>0$, we deduce that $G(x)>0$ for $x \in(0,1 / 3)$. The proof is complete. 
Lemma 5. There is a unique positive root $\eta_{m}$ in $(0,1 / 2)$ of the equation (2.4), and $\eta_{m}$ satisfies the inequality

$$
\eta_{m}^{2 m}+\frac{\eta_{m}}{1-\eta_{m}}+\frac{\eta_{m}^{m}}{\sqrt{1-\eta_{m}^{2 m}}} \leq 1
$$

Proof. We first prove the uniqueness of the solution in $(0,1 / 2)$ of the equation $(2.4)$.

Let $h(r)=r^{m}(2-3 r)+2 r-1$. Then it is easy to note that $h(0)=-1<0, h(1 / 2)=$ $\left(\frac{1}{2}\right)^{m+1}>0$ and, for $r \in[0,1 / 2]$, we have

$$
h^{\prime}(r)=m r^{m-1}(2-3 r)+2-3 r^{m}>0,
$$

showing that $h(r)$ is an increasing function of $r$ in [0,1/2], and thus, $h(r)=0$ has a unique root $\eta_{m}$ in $(0,1 / 2)$.

Now we verify the inequality (3.3). Let $y=\eta_{m}^{m}$. Then according to (2.4), we have $\eta_{m}=(1-2 y) /(2-3 y)$. Using this change of variables, we can rewrite $(3.3)$ in the following equivalent form:

$$
y^{2}+\frac{1-2 y}{1-y}+\frac{y}{\sqrt{1-y^{2}}} \leq 1 \text { for } y \in[0,1 / 2) .
$$

We note that

$$
y^{2}+\frac{1-2 y}{1-y}+\frac{y}{\sqrt{1-y^{2}}}-1=\frac{y\left[\left(-y^{2}+y-1\right) \sqrt{1-y^{2}}+1-y\right]}{(1-y) \sqrt{1-y^{2}}},
$$

and therefore, the inequality (3.3) is valid if and only if $\left(-y^{2}+y-1\right) \sqrt{1-y^{2}}+1-y \leq 0$ for $y \in[0,1 / 2)$, which holds if and only if $y^{2}\left(y^{3}-y^{2}+y+1\right) \geq 0$ for $y \in[0,1 / 2)$. Since the last inequality is obviously true, the proof is complete.

\section{Bohr-TYPE INEQUALITIES FOR BOUNDED ANALYTIC FUNCTIONS}

4.1. Proof of Theorem 1. Firstly, we consider the first part. Suppose that $f \in \mathcal{B}$, $a:=\left|a_{0}\right|$ and $\omega \in \mathcal{B}_{m}$. Then, by the classical Schwarz lemma and the Schwarz-Pick lemma or Lemma 1, we have

$$
\begin{aligned}
|\omega(z)| & \leq|z|^{m}, \quad z \in \mathbb{D} \\
|f(u)| & \leq \frac{|u|+a}{1+a|u|}, \quad u \in \mathbb{D},
\end{aligned}
$$

which implies

$$
|f(\omega(z))| \leq \frac{|\omega(z)|+a}{1+a|\omega(z)|} \leq \frac{r^{m}+a}{1+a r^{m}}, \quad|z|=r<1 .
$$

According to Lemma 3 and (4.3), we have

$$
B_{1}(f, r)+\frac{1+a r}{(1+a)(1-r)}\left\|f_{0}\right\|_{r}^{2} \leq \frac{\left(1-a^{2}\right) r}{1-r},
$$

and thus,

$$
A_{f}(z) \leq 1-\left[1-\frac{r^{m}+a}{1+a r^{m}}-\frac{\left(1-a^{2}\right) r}{1-r}\right]=1-\frac{(1-a) A_{m}(a, r)}{\left(1+a r^{m}\right)(1-r)}
$$


where $A_{m}(a, r)=(1-r)\left(1-r^{m}\right)-(1+a) r\left(1+a r^{m}\right)$, which is clearly a decreasing function of $a \in[0,1]$. Thus,

$$
A_{m}(a, r) \geq A_{m}(1, r)=(1-r)\left(1-r^{m}\right)-2 r\left(1+r^{m}\right),
$$

and obtain that $A_{f}(z) \leq 1$ if $A_{m}(1, r) \geq 0$, which holds for $r \leq \alpha_{m}$, where $\alpha_{m}$ is the unique positive root in $(0,1)$ of the equation $A_{m}(1, r)=0$.

To show that the radius $\alpha_{m}$ is best possible, we consider the functions

$$
\omega(z)=z^{m} \text { and } \varphi_{a}(z)=\frac{z+a}{1+a z}=a+\left(1-a^{2}\right) \sum_{n=0}^{\infty}(-a)^{n} z^{n+1}, a \in[0,1) .
$$

For the two functions, we get that (for $z=r$ )

$$
\begin{aligned}
A_{\varphi_{a}}(z) & =\left|\varphi_{a}\left(z^{m}\right)\right|+B_{1}\left(\varphi_{a}, r\right)+\frac{1+a r}{(1+a)(1-r)}\left\|\varphi_{a 0}-a\right\|_{r}^{2} \\
& =\frac{a+r^{m}}{1+a r^{m}}+\frac{\left(1-a^{2}\right) r}{1-a r}+\frac{\left(1-a^{2}\right)^{2} r^{2}}{(1+a)(1-r)(1-a r)} \\
& =\frac{r^{m}+a}{1+a r^{m}}+\frac{r\left(1-a^{2}\right)}{1-r},
\end{aligned}
$$

and this expression is bigger than 1 provided $r>\alpha_{m, a}$, where $\alpha_{m, a}$ is the unique positive root in $(0,1)$ of the equation $A_{m, a}(r)=0$. Allowing $a \rightarrow 1^{-}$gives that $\alpha_{m, 1}=\alpha_{m}$ is the best possible constant.

Next, we prove the second part. As in the previous case, by Lemma 3 and (4.3), it follows easily that

$$
B_{f}(z) \leq 1-\left[1-\left(\frac{r^{m}+a}{1+a r^{m}}\right)^{2}-\frac{\left(1-a^{2}\right) r}{1-r}\right]=1-\frac{\left(1-a^{2}\right) B_{m}(a, r)}{\left(1+a r^{m}\right)^{2}(1-r)},
$$

where $B_{m}(a, r)=(1-r)\left(1-r^{2 m}\right)-r\left(1+a r^{m}\right)^{2}$, which is clearly a decreasing function of $a \in[0,1]$. Thus,

$$
B_{m}(a, r) \geq B_{m}(1, r)=(1-r)\left(1-r^{2 m}\right)-r\left(1+r^{m}\right)^{2}=\left(1+r^{m}\right)\left(1-2 r-r^{m}\right)=: B_{m}(r) .
$$

We see that $B_{f}(z) \leq 1$ if $B_{m}(r) \geq 0$, which holds for $r \leq \beta_{m}$, where $\beta_{m}$ is the unique positive root in $(0,1)$ of the equation $B_{m}(r)=0$, namely, $(2.2)$ given by $1-2 r-r^{m}=0$.

To show the radius $\beta_{m}$ is best possible, we consider the functions $\omega(z)=z^{m}$ and $\varphi_{a}(z)$ as above, and find that (for $z=r$ )

$$
\begin{aligned}
B_{\varphi_{a}}(z) & =\left|\varphi_{a}\left(z^{m}\right)\right|^{2}+B_{1}\left(\varphi_{a}, r\right)+\frac{1+a r}{(1+a)(1-r)}\left\|\varphi_{a_{0}}-a\right\|_{r}^{2} \\
& =\left(\frac{r^{m}+a}{1+a r^{m}}\right)^{2}+\frac{r\left(1-a^{2}\right)}{1-r}
\end{aligned}
$$

and this expression is bigger than 1 provided $r>\beta_{m, a}$, where $\beta_{m, a}$ is the unique positive root in $(0,1)$ of the equation $B_{m, a}(r)=0$. Allowing $a \rightarrow 1^{-}$gives that $\beta_{m, 1}=\beta_{m}$ is the best possible constant. Thus the proof of Theorem 1 is complete. 
4.2. Proof of Theorem 2. We begin to recall from Lemma 2 that (as $\omega \in \mathcal{B}_{m}$ so that $|\omega(z)| \leq r^{m}$ and $f(\omega(0))=a_{0}$ with $\left.a=\left|a_{0}\right|\right)$,

$$
\left|f(\omega(z))-a_{0}\right| \leq \sum_{k=1}^{\infty}\left|a_{k}\right| r^{m k} \leq \begin{cases}r^{m} \frac{1-a^{2}}{1-r^{m} a} & \text { for } a \geq r^{m} \\ r^{m} \frac{\sqrt{1-a^{2}}}{\sqrt{1-r^{2 m}}} & \text { for } a<r^{m} .\end{cases}
$$

For the first part of the proof of the theorem, we first consider $a \geq r^{m}$. Then it follows from (4.1), the first inequality on the right of (4.5) and Lemma 3 that

$$
C_{f}(z) \leq a+\frac{r\left(1-a^{2}\right)}{1-r}+\frac{r^{m}\left(1-a^{2}\right)}{1-a r^{m}}=1-\frac{(1-a) C_{m}(a, r)}{\left(1-a r^{m}\right)(1-r)}
$$

where

$$
C_{m}(a, r)=r^{m+1} a^{2}-\left(r-3 r^{m+1}+2 r^{m}\right) a-\left(r^{m}-r^{m+1}+2 r-1\right) .
$$

Now, for fixed the value of $r$ in the expression, we obtain

$$
\frac{\partial C_{m}(a, r)}{\partial a}=r\left[2 a r^{m}-\left(1-3 r^{m}+2 r^{m-1}\right)\right] \leq\left.\frac{\partial C_{m}(a, r)}{\partial a}\right|_{a=1}=r\left[5 r^{m}-2 r^{m-1}-1\right],
$$

which is non-positive for $r \leq \nu_{m}, \nu_{m} \geq 3 / 5$, where $\nu_{m}$ is the unique root in $(0,1)$ of the equation $5 r^{m}-2 r^{m-1}-1=0$. So, $C_{m}(a, r)$ is a decreasing function of $a \in\left[r^{m}, 1\right]$ and thus, we have

$$
C_{m}(a, r) \geq C_{m}(1, r)=5 r^{m+1}-3 r^{m}-3 r+1=: C_{m}(r) .
$$

Clearly, $C(z) \leq 1$ if $C_{m}(r) \geq 0$, which holds for $r \leq \zeta_{m}$, where $\zeta_{m}$ is the unique positive root in $(0,1 / 3)$ of the equation $C_{m}(r)=0$ from Lemma 4 .

If $a<r^{m} \leq \zeta_{m}^{m}$, then combining (4.1), Lemma 3 and the second inequality on the right in (4.5), we have

$$
C_{f}(z) \leq a+\frac{r\left(1-a^{2}\right)}{1-r}+\frac{r^{m} \sqrt{1-a^{2}}}{\sqrt{1-r^{2 m}}}=: C(a, r)
$$

It is easy to see that

$$
\frac{\partial C(a, r)}{\partial r}=\frac{1-a^{2}}{(1-r)^{2}}+m r^{m-1} \sqrt{\frac{1-a^{2}}{\left(1-r^{2 m}\right)^{3}}}>0,
$$

showing that $C(a, r)$ is monotonically increasing with respect to $r \in\left[0, \zeta_{m}\right]$ for each fixed $a \in[0,1)$. Thus, we have from Lemma 4 that

$$
\begin{aligned}
C_{f}(z) & \leq a+\frac{\left(1-a^{2}\right) \zeta_{m}}{1-\zeta_{m}}+\frac{\zeta_{m}^{m} \sqrt{1-a^{2}}}{\sqrt{1-\zeta_{m}^{2 m}}} \\
& \leq \zeta_{m}^{m}+\frac{\zeta_{m}}{1-\zeta_{m}}+\frac{\zeta_{m}^{m}}{\sqrt{1-\zeta_{m}^{2 m}}} \leq 1
\end{aligned}
$$


To show the sharpness of the radius $\zeta_{m}$, we consider the functions $\omega$ and $\varphi_{a}$ as in (4.4), and obtain as before that (by setting $z=r$ for the first term in the definition of $C_{\varphi_{a}}(z)$ )

$$
C_{\varphi_{a}}(z)=a+\frac{r\left(1-a^{2}\right)}{1-r}+\frac{r^{m}\left(1-a^{2}\right)}{1-a r^{m}}
$$

and the last expression shows the radius $\zeta_{m}$ is optimal.

For the proof of the second part of the theorem, when $a \geq r^{m}$, it follows from (4.1), Lemma 3 and the first inequality on the right of (4.5) that

$$
D_{f}(z) \leq a^{2}+\frac{r\left(1-a^{2}\right)}{1-r}+\frac{r^{m}\left(1-a^{2}\right)}{1-a r^{m}}=1-\frac{\left(1-a^{2}\right) D_{m}(a, r)}{\left(1-a r^{m}\right)(1-r)}
$$

where

$$
D_{m}(a, r)=-a r^{m}(1-2 r)-r^{m}(1-r)-2 r+1 .
$$

For $r \leq 1 / 2$, it is clear that $D_{m}(a, r)$ is a decreasing function of $a, a \in\left[r^{m}, 1\right]$. Hence

$$
D_{m}(a, r) \leq D_{m}(1, r)=-r^{m}(2-3 r)-2 r+1=: D_{m}(r) .
$$

Obviously, $D_{f}(z) \leq 1$ if $D_{m}(r) \leq 0$, which holds for $r \leq \eta_{m} \leq 1 / 2$, where $\eta_{m}$ is the unique positive root in $(0,1 / 2]$ of the equation $D_{m}(r)=0$ from Lemma 5 .

If $a<r^{m} \leq \eta_{m}^{m}$, as in the previous case, we have

$$
D_{f}(z) \leq a^{2}+\frac{r\left(1-a^{2}\right)}{1-r}+\frac{r^{m} \sqrt{1-a^{2}}}{\sqrt{1-r^{2 m}}}=: D(a, r)
$$

Since $D(a, r)$ is clearly monotonically increasing with respect to $r \in\left[0, \eta_{m}\right]$, for each fixed value of $a$ in the expression, it follows from Lemma 5 that

$$
\begin{aligned}
D_{f}(z) & \leq a^{2}+\frac{\left(1-a^{2}\right) \eta_{m}}{1-\eta_{m}}+\frac{\eta_{m}^{m} \sqrt{1-a^{2}}}{\sqrt{1-\eta_{m}^{2 m}}} \\
& \leq \eta_{m}^{2 m}+\frac{\eta_{m}}{1-\eta_{m}}+\frac{\eta_{m}^{m}}{\sqrt{1-\eta_{m}^{2 m}}} \leq 1 .
\end{aligned}
$$

The sharpness part follows similarly. Thus, the proof of Theorem 2 is complete.

It is a simple exercise to see that for $0 \leq x \leq x_{0}(\leq 1)$, we have

$$
\Phi(x)=x+A\left(1-x^{2}\right) \leq \Phi\left(x_{0}\right) \text { whenever } 0 \leq A \leq 1 / 2,
$$

and similarly,

$$
\Psi(x)=x^{2}+A\left(1-x^{2}\right) \leq \Psi\left(x_{0}\right) \text { whenever } 0 \leq A \leq 1
$$

These two inequalities will be used in the proofs of Theorems 3 and 4 . 
4.3. Proof of Theorem 3. Firstly, we consider the first part. In view of (4.1), (4.3), Schwarz-Pick lemma and (3.1), we have

$$
\begin{aligned}
E_{f}(z) & \leq|f(w(z))|+\frac{r^{m}}{1-r^{2 m}}\left(1-|f(w(z))|^{2}\right)+\frac{\left(1-a^{2}\right) r^{2}}{1-r} \\
& \leq \frac{r^{m}+a}{1+a r^{m}}+\frac{r^{m}}{1-r^{2 m}}\left[1-\left(\frac{r^{m}+a}{1+a r^{m}}\right)^{2}\right]+\frac{\left(1-a^{2}\right) r^{2}}{1-r} \text { (by (4.6)) } \\
& =\frac{r^{m}+a}{1+a r^{m}}+\frac{r^{m}\left(1-a^{2}\right)}{\left(1+a r^{m}\right)^{2}}+\frac{\left(1-a^{2}\right) r^{2}}{1-r} \\
& =1+\frac{(1-a) E_{m}(a, r)}{\left(1+a r^{m}\right)^{2}(1-r)}
\end{aligned}
$$

for $r \in\left[0, \mu_{m}\right]$, since $\frac{r^{m}}{1-r^{2 m}} \leq \frac{1}{2}$ for $r \in\left[0, \mu_{m}\right]$, where $\mu_{m}=\sqrt[m]{\sqrt{2}-1}$ is the unique root in $(0,1)$ of the equation $r^{2 m}+2 r^{m}-1=0$, and

$$
\begin{aligned}
E_{m}(a, r)= & r^{2 m+2} a^{3}+r^{m+2}\left(r^{m}+2\right) a^{2}+\left[r^{2 m}(1-r)+r^{2}\left(2 r^{m}+1\right)\right] a \\
& +2 r^{m}(1-r)+r^{2}+r-1
\end{aligned}
$$

For each fixed $r \in[0,1]$, it is clear that $E_{m}(a, r)$ is a monotonically increasing function of $a \in[0,1)$ and thus,

$$
E_{m}(a, r) \leq E_{m}(1, r)=r^{m}\left(r^{m}+2\right)\left[2 r^{2}-r+1\right]+2 r^{2}+r-1=: E_{m}(r) .
$$

Therefore, $E_{f}(z) \leq 1$ if $E_{m}(r) \leq 0$, which is valid for $r \leq \gamma_{m}$, where $\gamma_{m}$ is the unique positive root in $(0,1)$ of the equation $E_{m}(r)=0$, and obviously $\gamma_{m}<\mu_{m}$.

To show that the radius $\gamma_{m}$ is optimal, as in the proofs of the previous two theorems, we consider the functions $\omega$ and $\varphi_{a}$ as in (4.4), and set $z=r$ for the first term in the definition of $E_{\varphi_{a}}(z)$ and obtain that

$$
\begin{aligned}
E_{\varphi_{a}}(z) & =\frac{a+r^{m}}{1+a r^{m}}+\frac{\left(1-a^{2}\right) r^{m}}{\left(1+a r^{m}\right)^{2}}+\frac{r^{2}\left(1-a^{2}\right)}{1-r} \\
& =1+\frac{(1-a) E_{m}(a, r)}{\left(1+a r^{m}\right)^{2}(1-r)},
\end{aligned}
$$

which is larger than 1 if and only if $E_{m}(a, r)>0$. Also, the expression on the right is smaller than or equal to 1 for all $a \in[0,1)$, only in the case when $r \leq \gamma_{m}$. Finally, it also suggests that $a \rightarrow 1$ in (4.8) shows that the expression on the right is larger than 1 if $r>\gamma_{m}$. 
Concerning the second sum in the theorem, it follows from (4.1), (4.3), Schwarz-Pick lemma and (3.1) that

$$
\begin{aligned}
F_{f}(z) & \leq|f(w(z))|^{2}+\frac{r^{m}}{1-r^{2 m}}\left(1-|f(w(z))|^{2}\right)+\frac{\left(1-a^{2}\right) r^{2}}{1-r} \\
& \leq\left(\frac{r^{m}+a}{1+a r^{m}}\right)^{2}+\frac{r^{m}}{1-r^{2 m}}\left[1-\left(\frac{r^{m}+a}{1+a r^{m}}\right)^{2}\right]+\frac{\left(1-a^{2}\right) r^{2}}{1-r} \\
& =\left(\frac{r^{m}+a}{1+a r^{m}}\right)^{2}+\frac{r^{m}\left(1-a^{2}\right)}{\left(1+a r^{m}\right)^{2}}+\frac{\left(1-a^{2}\right) r^{2}}{1-r} \\
& =1+\frac{\left(1-a^{2}\right) F_{m}(a, r)}{\left(1+a r^{m}\right)^{2}(1-r)},
\end{aligned}
$$

for $r \in\left[0, \tau_{m}\right]$, since $\frac{r^{m}}{1-r^{2 m}} \leq 1$ for $r \in\left[0, \tau_{m}\right]$, where $\tau_{m}=\sqrt[m]{\frac{\sqrt{5}-1}{2}}$ is the unique root in $(0,1)$ of the equation $r^{2 m}+r^{m}-1=0$, and

$$
F_{m}(a, r)=\left(r^{2 m}+r^{m}-1\right)(1-r)+r^{2}\left(1+a r^{m}\right)^{2} .
$$

Since $F_{m}(a, r)$ is clearly an increasing function of $a$ in $[0,1)$, it follows that

$$
F_{m}(a, r) \leq F_{m}(1, r)=r^{m}\left(r^{m}+1\right)\left[r^{m}-r+2\right]+r-1=: F_{m}(r) .
$$

Thus, $F_{f}(z) \leq 1$ if $F_{m}(r) \leq 0$, which holds for $r \leq \delta_{m}$, where $\delta_{m}$ is the unique positive root in $(0,1)$ of the equation $F_{m}(r)=0$, and obviously, $\delta_{m}<\tau_{m}$. Sharpness part may be proved similarly. Thus, we conclude the proof of the theorem.

4.4. Proof of Theorem 4. Firstly, we consider the first part. Clearly, $\frac{2 r}{1-r^{2 m}} \leq 1$ if $r \in\left[0, \xi_{m}\right]$, where $\xi_{m}$ is the unique root in $(0,1)$ of the equation $r^{2 m}+2 r-1=0$. As before, it follows from (4.1), (4.3), the Schwarz-Pick lemma and (3.1) that

$$
\begin{aligned}
G_{f}(z) & \leq|f(w(z))|+\frac{r}{1-r^{2 m}}\left(1-|f(w(z))|^{2}\right)+\frac{\left(1-a^{2}\right) r^{2}}{1-r} \\
& \leq \frac{r^{m}+a}{1+a r^{m}}+\frac{r}{1-r^{2 m}}\left[1-\left(\frac{r^{m}+a}{1+a r^{m}}\right)^{2}\right]+\frac{\left(1-a^{2}\right) r^{2}}{1-r} \\
& =\frac{r^{m}+a}{1+a r^{m}}+\frac{r\left(1-a^{2}\right)}{\left(1+a r^{m}\right)^{2}}+\frac{\left(1-a^{2}\right) r^{2}}{1-r} \\
& =1+\frac{(1-a) G_{m}(a, r)}{\left(1+a r^{m}\right)^{2}(1-r)},
\end{aligned}
$$

for $r \in\left[0, \xi_{m}\right]$, where

$$
\begin{aligned}
G_{m}(a, r)= & r^{2 m+2} a^{3}+r^{m+2}\left(r^{m}+2\right) a^{2}+\left[r^{2 m}(1-r)+r^{m+1}(2 r+1)+r\left(1-r^{m-1}\right)\right] a \\
& +r^{m}-r^{m+1}+2 r-1,
\end{aligned}
$$

which, for each fixed value of $r \in[0,1]$, is clearly monotonically increasing with respect to $a \in[0,1)$, because the coefficients of $a^{3}, a^{2}$ and $a$ are non-negative for $r \in[0,1]$. Hence

$$
G_{m}(a, r) \leq G_{m}(1, r)=2 r^{2 m+2}-r^{2 m+1}+r^{2 m}+4 r^{m+2}+3 r-1=: G_{m}(r) .
$$


Therefore, $G_{f}(z) \leq 1$ if $G_{m}(r) \leq 0$, which is valid for $r \leq \theta_{m}$, where $\theta_{m}$ is the unique positive root in $(0,1)$ of the equation $G_{m}(r)=0$, and it is clear that $\theta_{m}<\xi_{m}$.

To show that the radius $\theta_{m}$ is optimal, we consider the functions $\omega$ and $\varphi_{a}$ as in (4.4), and set $z=r$ for the first term in the definition of $G_{\varphi_{a}}(z)$ and obtain that

$$
\begin{aligned}
\left.G_{\varphi_{a}}(z)\right) & =\frac{a+r^{m}}{1+a r^{m}}+\frac{\left(1-a^{2}\right) r}{\left(1+a r^{m}\right)^{2}}+\frac{r^{2}\left(1-a^{2}\right)}{1-r} \\
& =1+\frac{(1-a) G_{m}(a, r)}{\left(1+a r^{m}\right)^{2}(1-r)}
\end{aligned}
$$

which is larger than 1 if $G_{m}(r)>0$, and using the earlier arguments, this is valid for $r>\theta_{m}$, where $\theta_{m}$ is the unique positive root in $(0,1)$ of the equation $G_{m}(r)=0$.

Obviously, $1-\frac{r}{1-r^{2 m}} \geq 0$ if $r \in\left[0, \chi_{m}\right]$, where $\chi_{m}$ is the unique root in $(0,1)$ of the equation $r^{2 m}+r-1=0$. Again, as in the previous case, we have for $r \in\left[0, \chi_{m}\right]$ that

$$
\begin{aligned}
H(z) & \leq|f(w(z))|^{2}+\frac{r}{1-r^{2 m}}\left(1-|f(w(z))|^{2}\right)+\frac{\left(1-a^{2}\right) r^{2}}{1-r} \\
& \left.\leq\left(\frac{r^{m}+a}{1+a r^{m}}\right)^{2}+\frac{r}{1-r^{2 m}}\left[1-\left(\frac{r^{m}+a}{1+a r^{m}}\right)^{2}\right]+\frac{\left(1-a^{2}\right) r^{2}}{1-r} \text { (by }(4.7)\right) \\
& \leq\left(\frac{r^{m}+a}{1+a r^{m}}\right)^{2}+\frac{r\left(1-a^{2}\right)}{\left(1+a r^{m}\right)^{2}}+\frac{\left(1-a^{2}\right) r^{2}}{1-r} \\
& =1+\frac{\left(1-a^{2}\right) H_{m}(a, r)}{\left(1+a r^{m}\right)^{2}(1-r)}
\end{aligned}
$$

for $r \in\left[0, \chi_{m}\right]$, where

$$
H_{m}(a, r)=(1-r)\left(r^{m}+r-1\right)+r^{2}\left(1+a r^{m}\right)^{2} .
$$

As $H_{m}(a, r)$ is an increasing function for $a$ in $[0,1)$, we obtain that

$$
H_{m}(a, r) \leq H_{m}(1, r)=r^{2 m+2}-r^{2 m+1}+r^{2 m}+2 r^{m+2}+2 r-1=: H_{m}(r) .
$$

Thus, $H(z) \leq 1$ is valid if $H_{m}(r) \leq 0$, which holds for $r \leq \vartheta_{m}$, where $\vartheta_{m}$ is the unique positive root in $(0,1)$ of the equation $H_{m}(r)=0$, and $\vartheta_{m}<\chi_{m}$ holds obviously. The sharpness part is similar. The proof of the theorem is complete.

4.5. Proof of Theorem 5. By Theorem E (and its proof), we only need to prove the case $m \geq 2$.

In fact, if $m \geq 2$ and $a \geq r^{m}$, then it follows from (4.1), Lemmas 2 and 3 that

$$
\begin{aligned}
I_{f}(z) & \leq a+\frac{r\left(1-a^{2}\right)}{1-r}+\left[\frac{|\omega(z)|\left(1-a^{2}\right)}{1-a|\omega(z)|}\right]^{2} \leq a+\frac{r\left(1-a^{2}\right)}{1-r}+\frac{r^{2 m}\left(1-a^{2}\right)^{2}}{\left(1-a r^{m}\right)^{2}} \\
& =1+\frac{(1-a) I_{m}(a, r)}{(1-r)\left(1-a r^{m}\right)^{2}}
\end{aligned}
$$


where

$$
\begin{aligned}
I_{m}(a, r)= & (2 r-1+a r)\left(1-2 a r^{m}+a^{2} r^{2 m}\right)+r^{2 m}(1-r)\left(1-a^{2}\right)(1+a) \\
= & -r^{3 m}(1-2 r) a^{3}-\left[r^{2 m}(2-3 r)+2 r^{m+1}\right] a^{2} \\
& +\left[r^{2 m}(1-r)+2(1-2 r) r^{m}+r\right] a+(1-r) r^{2 m}+2 r-1 .
\end{aligned}
$$

As

$$
\frac{\partial^{2} I_{m}(a, r)}{\partial a^{2}}=-6 r^{3 m}(1-2 r) a^{3}-2\left[r^{2 m}(2-3 r)+2 r^{m+1}\right] \leq 0 \text { for } r \leq 1 / 2,
$$

it follows that for $a \geq r^{m}$

$$
\frac{\partial^{2} I_{m}(a, r)}{\partial a^{2}} \leq\left.\frac{\partial^{2} I_{m}(a, r)}{\partial a^{2}}\right|_{a=r^{m}}=-6 r^{3 m}(1-2 r)-2 r^{2 m}(2-3 r)-4 r^{m+1} \leq 0,
$$

showing that $\frac{\partial I_{m}(a, r)}{\partial a}$ is a decreasing function of $a \in\left[r^{m}, 1\right]$. Thus, we obtain

$$
\frac{\partial I_{m}(a, r)}{\partial a} \geq\left.\frac{\partial I_{m}(a, r)}{\partial a}\right|_{a=1}=r\left(11 r^{2 m}-6 r^{2 m-1}-8 r^{m}+2 r^{m-1}+1\right),
$$

which is non-negative for $r \leq \xi_{m}$, where $\xi_{m} \approx 0.487478$ is the unique root in $(0,1)$ of the equation $11 r^{2 m}-6 r^{2 m-1}-8 r^{m}+2 r^{m-1}+1=0$. Therefore, $I_{m}(a, r)$ is clearly monotonically increasing with respect to $a \in\left[r^{m}, 1\right]$, and thus, we see that

$$
I_{m}(a, r) \leq I_{m}(1, r)=(3 r-1)\left(1-r^{m}\right)^{2} \leq 0 \text { for } r \leq 1 / 3 \text {. }
$$

We conclude that $I_{f}(z) \leq 1$ and hence, (2.9) holds, for $r \leq 1 / 3$ and $a \geq r^{m}$.

Next, we observe that $I_{m}(a, r)$ is an increasing function of $r \in[0,1)$ and may be written as

$$
\begin{aligned}
I_{m}(a, r)= & 1+\frac{1-a}{(1-r)\left(1-a r^{m}\right)^{2}}\left[\left(2 a^{3}+3 a^{2}-a-1\right) r^{2 m+1}-\left(a^{3}+2 a^{2}-a-1\right) r^{2 m}\right. \\
& \left.-\left(2 a^{2}+4 a\right) r^{m+1}+2 a r^{m}+(a+2) r-1\right] .
\end{aligned}
$$

Now, for $r \leq 1 / 3$, we have

$$
\begin{aligned}
I_{f}(z) & \leq I_{m}(a, 1 / 3)=1+\frac{1-a}{2\left(3^{m}-a\right)^{2}}\left[-a^{3}-3 a^{2}+2 a+2+3^{m}(1-a)\left(2 a-3^{m}\right)\right] \\
& \leq 1+\frac{1-a}{2\left(3^{m}-a\right)^{2}}\left[-a^{3}-3 a^{2}+2 a+2+3(1-a)(2 a-3)\right] \\
& =1+\frac{(1-a)^{2}}{2\left(3^{m}-a\right)^{2}}\left(a^{2}+10 a-7\right) \leq 1,
\end{aligned}
$$

since $a^{2}+10 a-7 \leq 0$ if $0 \leq a \leq a^{*}=-5+4 \sqrt{2}$. In the third inequality above we have used the fact that $3^{m}\left(2 a-3^{m}\right) \leq 3(2 a-3)$, i.e. $2 a-3^{m}-3 \leq 0$ for all $m \geq 1$.

Finally, if $m \geq 2$ and $0 \leq a<r^{m} \leq\left(\frac{1}{3}\right)^{m}$, then it follows from (4.1), Lemmas 2 and 3 that

$$
\begin{aligned}
I_{f}(z) & \leq a+\frac{r\left(1-a^{2}\right)}{1-r}+\left[\frac{|\omega(z)| \sqrt{1-a^{2}}}{\sqrt{1-|\omega(z)|^{2}}}\right]^{2} \\
& \leq a+\frac{r\left(1-a^{2}\right)}{1-r}+\frac{r^{2 m}\left(1-a^{2}\right)}{1-r^{2 m}}=: J_{m}(a, r) .
\end{aligned}
$$


We notice that $J_{m}(a, r)$ is monotonically increasing with respect to $r$ and thus, for $0 \leq$ $a<r^{m} \leq\left(\frac{1}{3}\right)^{m}$, we obtain that

$$
\begin{aligned}
I_{f}(z) & \leq J_{m}(a, 1 / 3)=a+\frac{1}{2}\left(1-a^{2}\right)+\frac{\left(\frac{1}{3}\right)^{2 m}\left(1-a^{2}\right)}{1-\left(\frac{1}{3}\right)^{2 m}} \\
& \leq\left(\frac{1}{3}\right)^{m}+\frac{1}{2}+\frac{\left(\frac{1}{3}\right)^{2 m}}{1-\left(\frac{1}{3}\right)^{2 m}} \\
& \leq \frac{1}{9}+\frac{1}{2}+\frac{1}{80}<1 .
\end{aligned}
$$

To show that the radius is optimal, we consider the functions $\omega$ and $\varphi_{a}$ as in (4.4), and set $z=r$ for the first term in the definition of $I_{\varphi_{a}}(z)$ and obtain that

$$
I_{\varphi_{a}}(z)=a+\frac{r\left(1-a^{2}\right)}{1-r}+\frac{r^{2 m}\left(1-a^{2}\right)^{2}}{\left(1-a r^{m}\right)^{2}},
$$

and the last expression easily delivers a proof of the sharpness part. The proof of Theorem 5 is complete.

Acknowledgments. This research of the first two authors are partly supported by Guangdong Natural Science Foundations (Grant No. 2018A030313508). The work of the third author was supported by Mathematical Research Impact Centric Support (MATRICS) of the Department of Science and Technology (DST), India (MTR/2017/000367). The authors of this paper thank the referees very much for their valuable comments and suggestions to this paper.

\section{REFERENCES}

1. Y. Abu-Muhanna, Bohr's phenomenon in subordination and bounded harmonic classes, Complex Var. Elliptic Equ. 55(11) (2010), 1071-1078.

2. Y. Abu-Muhanna and R. M. Ali, Bohr's phenomenon for analytic functions into the exterior of a compact convex body, J. Math. Anal. Appl. 379(2) (2011), 512-517.

3. Y. Abu-Muhanna and R. M. Ali, Bohr's phenomenon for analytic functions and the hyperbolic metric, Math. Nachr. 286(11-12) (2013), 1059-1065.

4. Y. Abu-Muhanna, R. M. Ali, Z. C. Ng, and S. F. M. Hasni, Bohr radius for subordinating families of analytic functions and bounded harmonic mappings, J. Math. Anal. Appl. 420(1) (2014), 124-136.

5. R. M. Ali, Y. Abu-Muhanna and S. Ponnusamy, On the Bohr inequality, In Progress in Approximation Theory and Applicable Complex Analysis, (Edited by N.K. Govil et al.), Springer Optimization and Its Applications, 117 (2016), 265-295.

6. R. M. Ali, R. W. Barnard and A. Yu. Solynin, A note on the Bohr's phenomenon for power series, J. Math. Anal. Appl. 449(1) (2017), 154-167.

7. S. A. Alkhaleefah, I. R. Kayumov and S. Ponnusamy, On the Bohr inequality with a fixed zero coefficient, Proc. Amer. Math. Soc. 147(12) (2019), 5263-5274.

8. C. Bénéteau, A. Dahlner and D. Khavinson, Remarks on the Bohr phenomenon, Comput. Methods Funct. Theory 4(1) (2004), 1-19.

9. B. Bhowmik and N. Das, Bohr phenomenon for subordinating families of certain univalent functions, J. Math. Anal. Appl. 462(2) (2018), 1087-1098.

10. H. Bohr, A theorem concerning power series, Proc. Lond. Math. Soc., 2(13) (1914), 1-5.

11. A. Defant, L. Frerick, J. Ortega-Cerdà, M. Ounaïes, and K. Seip, The Bohnenblust-Hille inequality for homogenous polynomials is hypercontractive, Ann. of Math. 174(2) (2011), 512-517. 
12. P. B. Djakov and M. S. Ramanujan, A remark on Bohr's theorems and its generalizations, J. Analysis 8 (2000), 65-77.

13. S. R. Garcia, J. Mashreghi and W. T. Ross, Finite Blaschke products and their connections, Springer, Cham, 2018.

14. I. R. Kayumov and S. Ponnusamy, Bohr inequality for odd analytic functions, Comput. Methods Funct. Theory, 17 (4)(2017), 679-688.

15. I. R. Kayumov and S. Ponnusamy, Bohr's inequality for analytic functions with lacunary series and harmonic functions, J. Math. Anal. and Appl., 465(2)(2018), 857-871.

16. I. R. Kayumov and S. Ponnusamy, Improved version of Bohr's inequality, C. R. Math. Acad. Sci. Paris 356(3) (2018), 272-277

17. A. A. Ismagilov, A. V. Kayumova, I. R. Kayumov and S. Ponnusamy, Bohr inequalities in some classes of analytic functions. (Russian) Complex analysis (Russian), 69-83, Itogi Nauki Tekh. Ser. Sovrem. Mat. Prilozh. Temat. Obz., 153, Vseross. Inst. Nauchn. i Tekhn. Inform. (VINITI), Moscow, 2018.

18. A. Kayumova, I. R. Kayumov and S. Ponnusamy, Bohr's inequality for harmonic mappings and beyond, Mathematics and computing, 245-256, Commun. Comput. Inf. Sci., 834, Springer, Singapore, 2018.

19. I. R. Kayumov, S. Ponnusamy and N. Shakirov, Bohr radius for locally univalent harmonic mappings, Math. Nachr. 291 (2017), 1757-1768.

20. G. Liu and S. Ponnusamy, On Harmonic $\nu$-Bloch and $\nu$-Bloch-type mappings, Results Math. 73(3)(2018), Art 90, 21 pages;

21. G. Liu, Z.H. Liu and S. Ponnusamy, Refined bohr inequality for bunded analytic functions, Preprint, 9 pages; Preprint in: arXiv:2006.08930v1

22. M. S. Liu, Y. M. Shang and J. F. Xu, Bohr-type inequalities of analytic functions, J. Inequal. Appl., 345 (2018), 13 pp.

23. M. S. Liu, S. Ponnusamy and J. Wang, Bohr's phenomenon for the classes of Quasi-subordination and $K$-quasiregular harmonic mappings, RACSAM 114, 115 (2020). https://doi.org/10.1007/s13398020-00844-0

24. Z. H. Liu and S. Ponnusamy, Bohr radius for subordination and $K$-quasiconformal harmonic mappings, Bull. Malays. Math. Sci. Soc., 42 (2019), 2151-2168.

25. V. I. Paulsen, G. Popascu, and D. Singh, On Bohr's inequality, Proc. Lond. Math., 85(2) (2002), 493-512.

26. V. I. Paulsen and D. Singh, Bohr's inequality for uniform algebras, Proc. Amer. Math. Soc., 132 (2004), 3577-3579.

27. V. I. Paulsen and D. Singh, Extensions of Bohr's inequality, Bull. Lond. Math. Soc., 38(6) (2006), 991-999.

28. S. Ponnusamy, R. Vijayakumar and K.-J. Wirths, New inequalities for the coefficients of unimodular bounded functions, Results Math. (2020), 75:107, 11 pages; https://doi.org/10.1007/s00025-02001240-1

29. S. Ponnusamy and K.-J. Wirths, Bohr type inequalities for functions with a multiple zero at the origin, Comput. Methods Funct. Theory (2020). https://doi.org/10.1007/s40315-020-00330-z

30. S. Sidon, Über einen Satz von Herrn Bohr, Math. Z. 26(1) (1927), 731-732.

31. M. Tomić, Sur un théorème de H. Bohr, Math. Scand. 11 (1962), 103-106. 
Y. Huang, School of Mathematical Sciences, South China Normal University, Guangzhou, GuANGDONG 510631, China.

E-mail address: hyong95@163.com

M-S Liu, School of Mathematical Sciences, South China Normal University, Guangzhou, Guangdong 510631, China.

E-mail address: liumsh65@163.com

S. Ponnusamy, Department of Mathematics, Indian Institute of Technology Madras, Chennai-600 036, India.

E-mail address: samy@iitm.ac.in 